\title{
Review Article \\ Recent Advances in Wireless Indoor Localization Techniques and System
}

\author{
Zahid Farid, Rosdiadee Nordin, and Mahamod Ismail \\ School of Electrical, Electronics \& System Engineering, University Kebangsaan Malaysia (UKM), 43600 Bangi, \\ Selangor, Malaysia
}

Correspondence should be addressed to Zahid Farid; zahidf9@yahoo.com

Received 17 May 2013; Accepted 17 August 2013

Academic Editor: Rui Zhang

Copyright (c) 2013 Zahid Farid et al. This is an open access article distributed under the Creative Commons Attribution License, which permits unrestricted use, distribution, and reproduction in any medium, provided the original work is properly cited.

The advances in localization based technologies and the increasing importance of ubiquitous computing and context-dependent information have led to a growing business interest in location-based applications and services. Today, most application requirements are locating or real-time tracking of physical belongings inside buildings accurately; thus, the demand for indoor localization services has become a key prerequisite in some markets. Moreover, indoor localization technologies address the inadequacy of global positioning system inside a closed environment, like buildings. Based on this, though, this paper aims to provide the reader with a review of the recent advances in wireless indoor localization techniques and system to deliver a better understanding of state-of-the-art technologies and motivate new research efforts in this promising field. For this purpose, existing wireless localization position system and location estimation schemes are reviewed, as we also compare the related techniques and systems along with a conclusion and future trends.

\section{Introduction}

Location based services (LBSs) [1] are a significant permissive technology and becoming a vital part of life. In this era, especially in wireless communication networks, LBS broadly exists from the short-range communication to the long-range telecommunication networks. LBS refers to the applications that depend on a user's location to provide services in various categories including navigation, tracking, healthcare, and billing. However, its demand is increasing with new ideas with the advances in the mobile phone market. The core of the LBSs is positioning technologies to find the motion activity of the mobile client. After detection, we pass these statistics to the mobile client on the move at the right time and the right location. So, the positioning technologies have a major influence on the performance, reliability, and privacy of LBSs, systems, and applications [2].

The basic components of LBS are software application (provided by the provider), communication network (mobile network), a content provider, a positioning device, and the end user's mobile device. There are several ways to find the location of a mobile client indoors and outdoors. The most popular technology outdoors is global positioning system (GPS) [1]. Location finding refers to a process of obtaining location information of a mobile client (MC) with respect to a set of reference positions within a predefined space. In the literature, many terms are used for location finding like position location, geolocation, location sensing, or localization [3]. Position system is a system arranged in such a way to find or estimate the location of an object. The aims of this paper are to provide the reader with fingerprinting based wireless indoor localization techniques and systems for indoor applications. The authors hope that this paper will benefit researchers working in this field, users, and developers in terms of using these systems and will help them identify the potential research shortcoming and future application products in this emerging area.

1.1. Indoor versus Outdoor Positioning. Positioning system can be categorized depending on the target environment as either indoor, outdoor, or mixed type. For localization in an outdoor environment, global navigation satellite systems (GNSS) such as GPS have been used in a wide range 
of applications including tracking and asset management systems; transport navigation and guidance; synchronization of telecommunications networks; geodetic survey. GPS works extremely well in outdoor positioning. Unfortunately, GPS does not perform well in urban canyons, close to walls, buildings, trees, indoors, and in underground environments as the signal from the GPS satellites is too weak to come across most buildings thus making GPS ineffective for indoor localization [4].

Indoor positioning [5] can be defined as any system that provides a precise position inside of a closed structure, such as a shopping mall, hospitals, airport, a subway, and university campuses. By the complex nature of indoor environments, the development of an indoor localization technique is always associated with a set of challenges like smaller dimensions, high none line of sight (NLOS), influence of obstacles like walls, equipment, movement of human beings, doors, and other factors. Multipath effect signals are reflected and attenuated by walls and furniture and noise interference [6]. These challenges result mainly from the influence of obstacles on the propagation of electromagnetic waves. For getting good and accurate results, a positioning system must be able to handle these problems. Beside this, higher accuracy is also required indoors to locate a user at least in the right room. One of the important aspects indoors is indoor signal property characteristics. A signal strength pattern around an access point interference is shown in Figure 1. Some of the current indoor navigation technologies are listed in Figure 2.

\section{Performance Metrics}

The performance criteria associated with localization systems can be classified into the following areas.

2.1. Accuracy. Accuracy (or location error) of a system is the important user requirement of positioning systems. Accuracy can be reported as an error distance between the estimated location and the actual mobile location. Sometimes, accuracy is also called the area of uncertainty; that is, the higher the accuracy is, the better the system is.

2.2. Responsiveness. The responsiveness determines how quickly the location estimate of a moving target is updated.

2.3. Coverage. The problem of determining the network coverage for a designated area is important when evaluating the effectiveness of a positioning system. Coverage is closely related to accuracy. Coverage can be categorized as local coverage, scalable coverage, and global coverage. Local coverage is a small well-defined, limited area which is not extendable (e.g., a single room or building). In this case, the coverage size is specified (e.g., $(\mathrm{m}),\left(\mathrm{m}^{2}\right)$, or $\left.\left(\mathrm{m}^{3}\right)\right)$. Scalable coverage means systems with the ability to increase the area by adding hardware, and global coverage means system performance worldwide or within the desired/specified area.

2.4. Adaptiveness. Environmental influence changes may affect the localization system performance. The ability of the

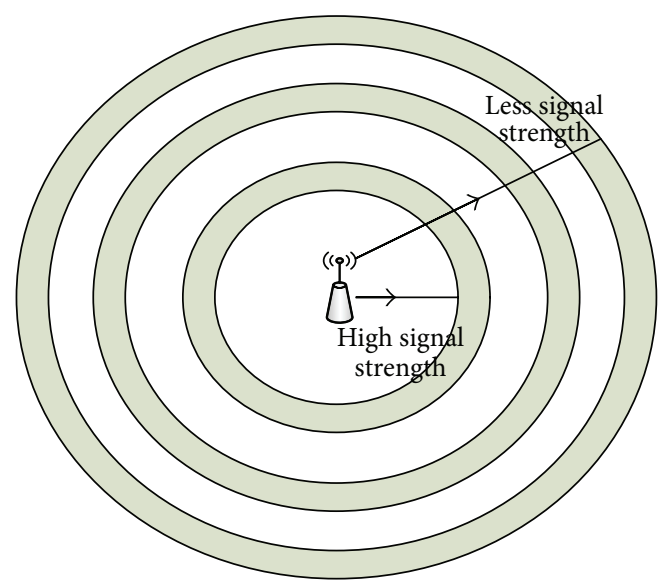

FIGURE 1: Signal strength pattern.

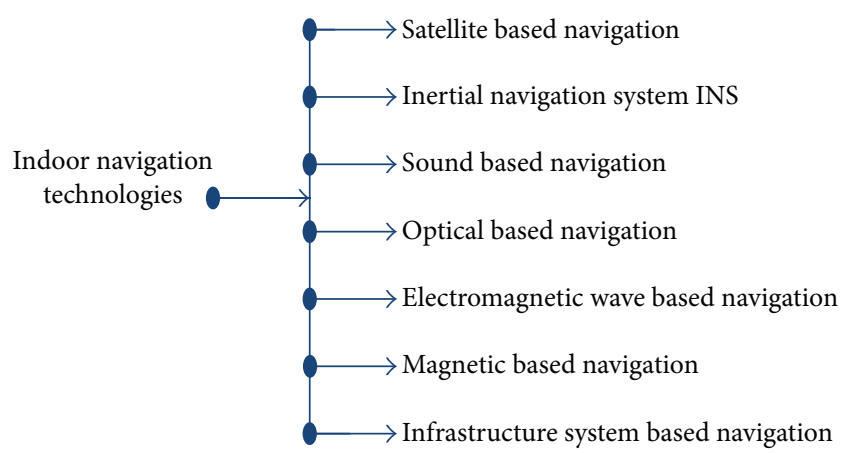

FIGURE 2: Indoor navigation technologies.

localization system to cope with these changes is called its adaptiveness. A system that is able to adapt to environmental changes can provide better localization accuracy than systems that cannot adapt. An adaptive system can also prevent the need for repeated calibration.

2.5. Scalability. Scalability is a desirable property in almost any system that suggests how well the system performs when it operates with a larger number of location requests and a larger coverage. Poor scalability can result in poor system performance, necessitating the reengineering or duplication of systems. A scalable positioning system should be able to handle large numbers of tags without unnecessary strain.

2.6. Cost and Complexity. The cost gained from a positioning system can arise from the cost of extra infrastructure, additional bandwidth, money, lifetime, weight, energy, and nature of deployed technology. The cost may include installation and survey time during the deployment period. If a positioning system can reuse an existing communication infrastructure, some part of infrastructure, equipment, and bandwidth can be saved. The complexity of the signal processing and algorithms used to estimate the location is another issue that needs to be balanced with the performance of positioning 
systems. Tradeoffs between the system complexity and the accuracy affect the overall cost of the system.

\section{Location Detection Techniques and Location Algorithms}

Several different methods are used for location techniques and algorithms in wireless based localization. Location detection techniques can be divided into three general categories: proximity, triangulation and scene analysis as shown in Figure 3.

\subsection{Proximity Detection (Connectivity Based Positioning).} Proximity detection or connectivity based is one of the simplest positioning methods to implement. It provides symbolic relative location information. The position of mobile client is determined by cell of origin $(\mathrm{CoO})$ method with known position and limited range [7]. When more than one beacon detects the mobile target, it simply forwards the position nearest where the strongest signal is received. The accuracy of $\mathrm{CoO}$ relates to the density of beacon point deployment and signal range. This method is implemented with several wireless positioning technologies, in particular, the system running infrared radiation (IR), radio frequency identification (RFID) GSM (Cell-ID), bluetooth, and custom radio devices [3].

3.2. Triangulation. Triangulation uses the geometric properties of triangles to determine the target location. It has two derivations: lateration and angulation. Techniques based on the measurement of the propagation-time system (e.g., TOA, RTOF, and TDOA) and RSS-based and received signal phase methods are called lateration technique $[8,9]$. The AOA estimation technique is also called an angulation technique.

\subsubsection{Angle Based Method}

Angle of Arrival (AoA)/Angulation. The angle of arrival (AoA) technique determines the angle of arrival of the mobile signal coming from a known location at which it is received at multiple base stations [3]. To estimate position in a $2 \mathrm{D}$ dimension plane, AoA approach requires only two beacons. To improve accuracy, three beacons or more are used for location estimation (triangulation). For finding direction, it requires highly directional antennas or antenna arrays. Geometric relationships can then be used to estimate the location of the intersection of two lines of bearing (LoBs) from the known reference points as shown in Figure 4.

AOA-based techniques have their limitations. AOA requires additional antennas with the capacity to measure the angles which increase the cost of the AOA system implementation. In indoor environments, AOA-based methods are affected by multipath and NLOS propagation of signals, along with reflections from walls and other objects, so it is not good for indoor implementation. Due to these factors, it can significantly change the direction of signal arrival and thus degrade the accuracy of an indoor AOA-based positioning system [10].

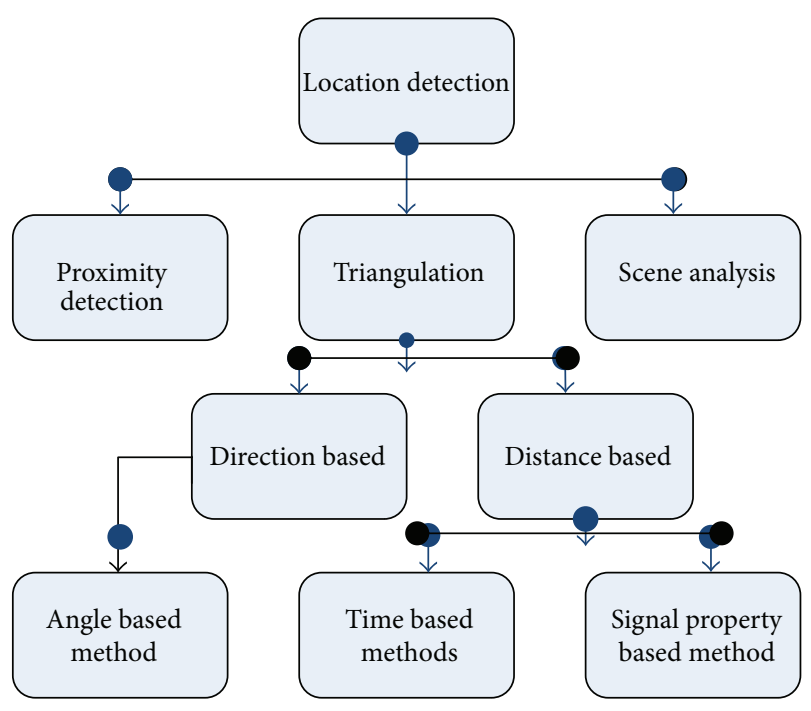

FIGURE 3: Location detection based classification.

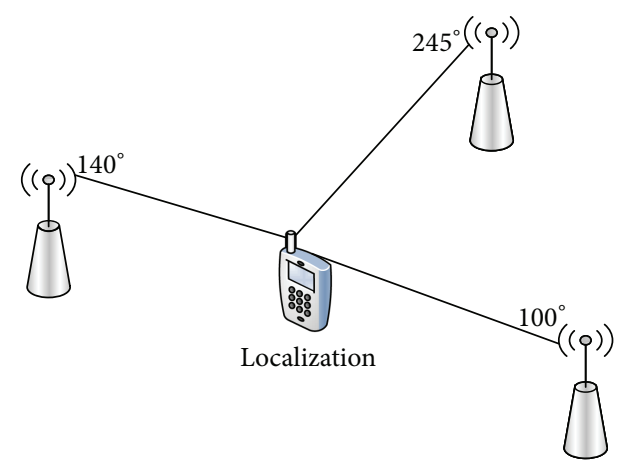

FIGURE 4: Angle-of-arrival positioning method.

\subsubsection{Time Based Methods}

Lateration/Trilateration/Multilateration. All three terms (lateration/trilateration/multilateration) refer to a position determined from distance measurements. Lateration or trilateration determines the position of an object by measuring its distance from multiple reference points. Thus, it is also called range measurement technique. In trilateration, the "tri" says that at least three fixed points are necessary to determine a position. Techniques based on the measurement of the propagation-time system (e.g., TOA, RTOF, and TDOA) and RSS-based and received signal phase methods are called lateration techniques $[5,11]$.

Time of Arrival (ToA)/Time of Flight (ToF). Time of Arrival (ToA) systems are based on the accurate synchronization of the arrival time of a signal transmitted from a mobile device to several receiving beacons as shown in Figure 5. In ToA, the mobile device transmits a time stamped signal towards receiving beacons. When it is received, the distance between the mobile node and the receiving beacons is calculated from the transmission time delay and the corresponding speed of the signal. 


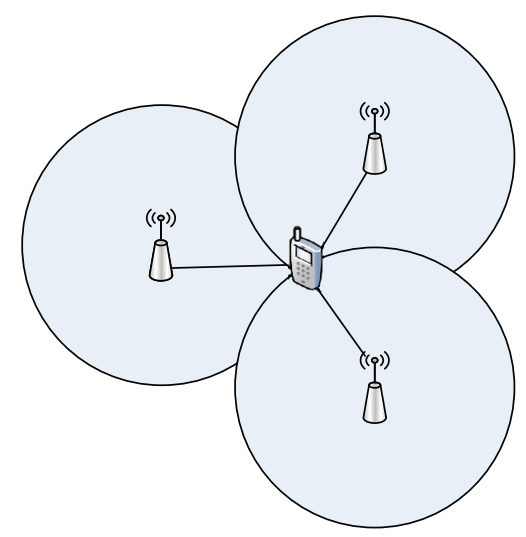

FIgURE 5: Positioning based on TOA/RTOF measurements.

ToA method needs precise knowledge of the transmission start time(s). Due to this, all receiving beacons along with mobile devices are accurately synchronized with a precise time source. ToA is the most accurate technique used in indoor environment which can filter out multi-path effects [4]. One of the disadvantages of TOA approach is the requirement for precise time synchronization of all the devices. For time delay measurement, an additional server will be needed which will increase the cost of the system. Along with this, increased delay can also be propagated by a denser environment, in terms of more people.

Time Difference of Arrival (TDoA). Time Difference of Arrival (TDoA) techniques are measured between multiple pairs of reference points with known locations and use relative time measurements at each receiving node in place of absolute time measurements illustrated in Figure 6. TDoA does not need the use of a synchronized time source of transmission in order to resolve timestamps and find the location. With TDoA, a transmission with an unknown starting time is received at various receiving nodes, with only the receivers requiring time synchronization [5]. Each difference of arrival time measurement produces a hyperbolic curve in the localization space on which the location of the mobile node lies. The intersection of multiple hyperbolic curves specifies the possible locations of the client. Localization using TDOA is called multilateration.

Round Trip Time (RTT)/Round-Trip Time of Flight (RToF). It measures the time of flight of the signal pulse traveling from the transmitter to the measuring unit and back [3]. In TOA, calculating the delay is by using two local clocks in both nodes, while in RTT, it uses only one node to record the transmitting and arrival times. Because of this advantage, this technology solves the problem of synchronization to some extent. One of the drawbacks of this method is range measurements to multiple devices that need to be carried out consecutively which may cause precarious latencies for applications where devices move quickly.

3.2.3. Signal Property Based Method. The majority of wireless localization systems compute the distance to the positioning

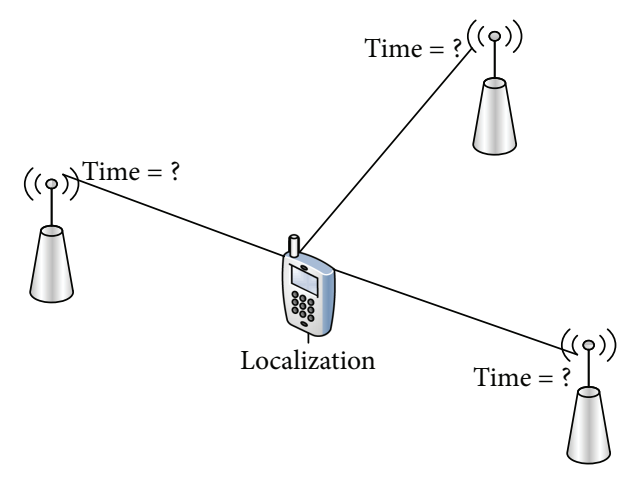

Figure 6: Time difference of arrival (source [31]). device using either timing information or angle based. In both scenarios, they are influenced by the multipath effect. Due to this, the accuracy of estimated location can be decreased. The substitute method is to estimate the distance of unknown node to reference node from some sets of measuring units using the attenuation of emitted signal strength $[3,12]$. This method can only be possible with radio signals. Mostly wireless localization systems positioning device using properties of the received signal, with received signal strength indicator (RSSI) being the most widely used signal-related feature. RSSI measurement estimations depend heavily on the environmental interference, and they are also nonlinear. These methods work with the WiFi technology. As this system needs a server for implementation, this technique can work using only access points which are cheaper than Wi-Fi routers.

3.3. Dead Reckoning (DR). Dead reckoning is the process of estimating known current position based on last determined position and incrementing that position based on known or estimated speeds over elapsed time. An inertial navigation system which provides very accurate directional information uses dead reckoning and is very widely applied [13]. One of the disadvantages of dead reckoning is that the inaccuracy of the process is cumulative, so the deviation in the position fix grows with time. The reason is that new positions are calculated entirely from previous positions. Research has been carried out $[13,14]$ in indoor localization using dead reckoning.

3.4. Map Matching (MM). This method is based on the theory of pattern recognition [15] which combines electronic map with locating information to obtain the real position of vehicles in a road network. The use of maps is an efficient alternative to the installation of additional hardware. MM techniques include topological analyses, pattern recognition, or advanced techniques such as hierarchical fuzzy inference algorithms. The authors of $[16,17]$ present a recent research work on map matching algorithm based on indoor and LBS, respectively. 


\section{Position Systems}

This section presents a review of most prominent state-ofthe-art wireless positioning systems as shown in Figure 7. Our main focus is put on the radio based systems especially in wireless local area network (WLAN) positioning (Table 1).

Global positioning system (GPS) is the most popular and worldwide radio navigation system to find the location and the position of the objects especially for outdoor environment [18]. However, it does not work well in indoor setups because the presence of obstacles in the line of sight between the satellite and the receiver and electromagnetic waves is spread and attenuated by the buildings and outdoor obstacles [8]. As people spend most of their time in a closed environment (indoors), GPS is not well suited for indoor positioning tracking (Table 3).

Infrared radiation (IR) positioning systems are one of the most common positioning systems that use wireless technology. The spectral region of infrared has been used in various ways for detection or tracking of objects or persons and available in various wired and wireless devices such as mobile phone, PDAs, and TV [19]. Most IR based wireless devices uses line-of-sight (LOS) communication mode between transmitter and receiver without interference from strong light sources [20]. The main advantage of using IR based system devices is being small, lightweight, and easy to carry out. The IR systems undertake an indoor positioning determination in a precise way. Besides these, IR based indoor positioning systems have some disadvantages like security and privacy issues. IR signals have some limitations for location determination, like interference from fluorescent light and sunlight [4]. Beside this, the IR based indoor system has expensive system hardware and maintenance cost.

Radio frequency technologies [21] are commonly used in location position systems because of some advantages; for example, radio waves can penetrate through obstacles like building walls and human bodies easily. Due to this, the positioning system in RF based has a larger coverage area and needs less hardware comparing to other systems. In addition, RF based technologies are further divided into narrow band based technologies (RFID, bluetooth, WLAN, and FM) and wide band based technologies (UWB) [19]. RADAR [22] by Microsoft Research was the first RF based technique for location determination and user tracking.

Radio frequency identification (RFID) has been recognized as the next promising technology in serving the positioning system for locating objects or people. RFID enables a one way wireless communication using a noncontact and advanced automatic identification technology that uses radio signals that put an RFID tag on people or objects, for the purpose of automatic identification, tracking, and management. Tracking the movements of objects in RFID is done through a network of radio enabled scanning devices over a distance of several meters. RFID technology is used in a wide range of applications including people, automobile assembly industry, warehouse management, supply chain network, and assets without the need of line of sight contact [9].

Bluetooth is a wireless standard for wireless personal area networks (WPANs). Almost every WiFi enabled mobile device, such as mobile phone or computer, also has an embedded bluetooth module. Bluetooth operates in the $2.4 \mathrm{GHz}$ ISM band. The benefit of using bluetooth for exchanging information between devices is that this technology is of high security, low cost, low power, and small size. Each bluetooth tag has a unique ID, which can be used for locating the Bluetooth tag. There are several recent research works dedicated to bluetooth based localization systems [23, 24]. One of the drawbacks of using bluetooth technology in localization is that, in each location finding, it runs the device discovery procedure; due to this, it significantly increases the localization latency (10-30 s) and power consumption as well. That is why bluetooth device has a latency unsuitable for realtime positioning applications.

Ultrawideband (UWB) is a radio technology for shortrange, high-bandwidth communication holding the properties of strong multipath resistance. Widespread use of UWB in a variety of localization applications requiring higher accuracy $20-30 \mathrm{~cm}$ than achievable through conventional wireless technologies (e.g., radio frequency identification (RFID), wireless local area networks (WLAN), etc.) [25]. A typical UWB setup structures stimulus radio wave generator and receivers which capture the propagated and scattered wave. Moreover, UWB hardware is expensive, making it costly for wide-scale use.

The FM radio based system is popular through the ages. It is widely available across the globe especially in most households and in cars. FM radio uses the frequency-division multiple access (FDMA) approach which splits the band into a number of separate frequency channels that are used by stations. FM band ranges and channel separation distances vary in different regions. There are only a few works dedicated to FM radio based positioning. Recently [26, 27] presented research that worked on indoor positioning using FM radio signals.

The ZigBee technology is an emerging wireless technology standard which provides solution for short and medium range communications due to its numerous benefits [28]. It is mainly designed for applications which require low-power consumption but do not require large data throughput. The signal range coverage of a ZigBee in indoor environments is typically $20 \mathrm{~m}$ to $30 \mathrm{~m}$. Distance calculation between two ZigBee nodes is usually carried out from RSSI values. ZigBee is open to interference from a wide range of signal types using the same frequency which can disrupt radio communication because it operates in the unlicensed ISM bands. Hu et al. [7] deployed a ZigBee based localization algorithm for indoor environments. Beside this, Fernández et al. [29] proposed a way to improve the position determination in an indoor location system (ILS) based on the power levels (RSSI) of an ad hoc ZigBee network.

Hybrid positioning systems are defined as systems for determining the location of a mobile client combining several different positioning technologies [30]. Many location technologies are used to estimate the position of mobile client in a space or grid, based on some mathematical models. The local positioning systems fail to work outdoors, whereas the GPS based positioning systems do not work inside buildings due to the absence of line of sight to the satellites. So, there 


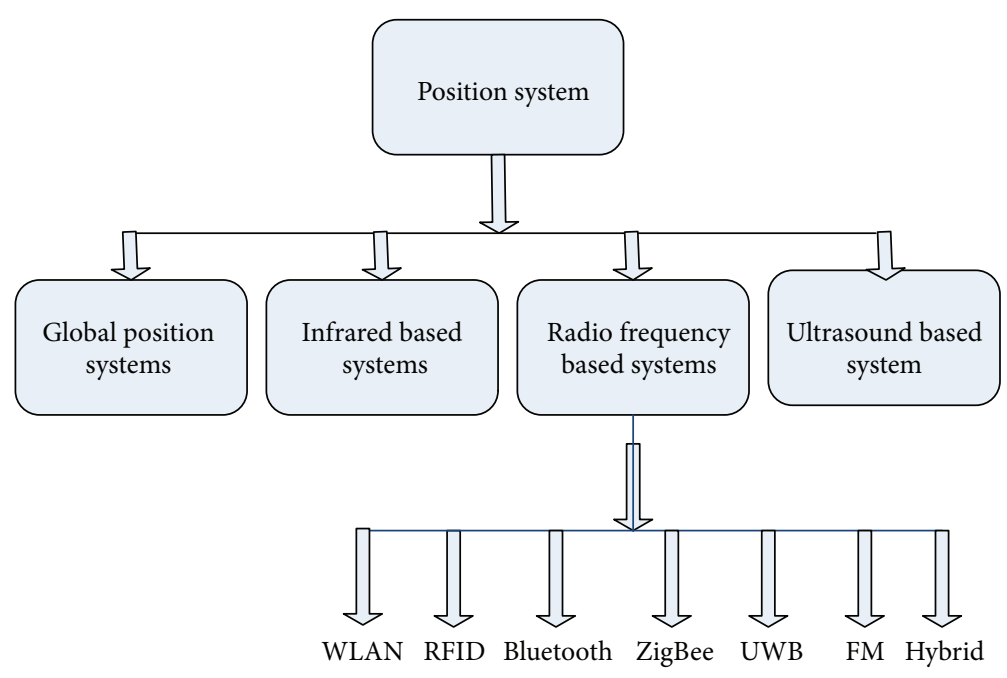

FIgURE 7: Taxonomy of position systems.

is a need for positioning systems that can work both indoors and outdoors, and hence, the concept of hybrid positioning systems is used [31]. Different hybrid positioning systems are currently being developed and used in services from Combain Mobile, Navizon, Xtify, PlaceEngine, SkyHook, Devicescape, Google Maps for Mobile, and sopenBmap for application in smartphones.

Ultrasound system is a technology based on the nature of bats and operates in the low frequency band compared to the other two signaling technologies. The ultrasound signals are used to estimate the position of the emitter tags from the receivers. Ultrasound is unable to penetrate walls but reflects off most of the indoor obstructions. However, it has a lower level of accuracy (in centimeters) and suffers a lot of interference from reflected ultrasound signals propagated around by other sources such as the collision of metals. Some recent research work $[32,33]$ was carried out under ultrasound based indoor localization.

4.1. WiFi-Based Indoor Localization. One of the advantages of using WiFi Positioning Systems is to locate the position of almost every WiFi compatible device without installing extra software or manipulating the hardware. Beside this, in WLAN, line of sight is not required. Due to this advantage, WiFi positioning systems have become the most widespread approach for indoor localization [11].

Most positioning systems based on WLAN (WiFi) are available as commercial products as prototypes based on measurements on the received signal strength (RSS). WiFi based positioning systems have several advantages.

Firstly in terms of cost effect, WLAN infrastructures implementation of position algorithms does not need any additional hardware as network interface cards (NICs) measure signal strength values from all wireless access points in range of the receiver. Therefore, signals needed for positioning can be obtained directly from NICs available on most handheld computing devices. Due to the ubiquity of WLANs, this mode of positioning provides a particularly cost-effective solution for offering LBS in commercial and residential indoor environments [34]. Secondly, WLAN positioning systems offer scalability in two respects: first, no costly requirement of infrastructure and hardware and second the number of mobile devices subscribing to positioning services. Beside this, there are also certain WLAN limitations: signal attenuation of the static environment like wall, movement of furniture and doors. Some of the WiFi strengths, weaknesses, and opportunities are presented in Table 2.

4.2. Fingerprinting Based Indoor Localization. Most indoor localization approaches adopted fingerprint matching as the basic scheme of location determination. The main theme is to collect features of the scene (fingerprint) from the surrounding signatures at every location in the areas of interest and then build a fingerprint database. The location of an object is then determined by matching online measurement with the closed location against the database [35] (Figure 8).

This method does not require specialized hardware in either the mobile device or the receiving end nor is no time synchronization necessary between the stations. It may be implemented totally in software which can reduce complexity and cost significantly compared to angulation or purely timebased lateration systems [34].

The location fingerprinting also called a fingerprinting method consists of two phases [5]. Phase 1 is the so-called calibration phase, offline phase, or training phase, and phase 2 is the localization phase or online phase. In the offline phase, maps for fingerprinting are set up either empirically in measurement operations or computed analytically (signal strength reference values (anchor point) can be computed using a signal propagation model). In the first phase, a creation of radio maps for site survey where the positioning is supposed to work must be recorded. Basically, radio map is a database of spots at predefined points (coordinates) coupled with various radio signal characteristics, for example, RSS, signal angles, or propagation time called signal fingerprints. Step by step, for every fingerprint, there must 
TABLE 1: Comparisons of indoor position methods.

\begin{tabular}{|c|c|c|c|c|c|c|c|}
\hline Method & $\begin{array}{l}\text { Measurement } \\
\text { type }\end{array}$ & $\begin{array}{l}\text { Indoor } \\
\text { accuracy }\end{array}$ & Coverage & $\begin{array}{l}\text { Line of sight } \\
\text { (LOS)/nonline- } \\
\text { of sight } \\
\text { (NLOS) }\end{array}$ & $\begin{array}{l}\text { Affected } \\
\text { by } \\
\text { multipath }\end{array}$ & Cost & Notes \\
\hline Proximity & Signal type & $\begin{array}{l}\text { Low to } \\
\text { high }\end{array}$ & Good & Both & No & Low & $\begin{array}{l}\text { (1) Accuracy can be improved by using } \\
\text { additional antenna. However, it will } \\
\text { increase the cost. } \\
\text { (2) Accuracy is on the order of the size } \\
\text { of the cells. }\end{array}$ \\
\hline Direction (AoA) & $\begin{array}{l}\text { Angle of } \\
\text { arrival }\end{array}$ & Medium & $\begin{array}{l}\text { Good } \\
\text { (Multipath } \\
\text { issues) }\end{array}$ & LOS & Yes & High & $\begin{array}{l}\text { (1) Accuracy depends on the antenna's } \\
\text { angular properties. } \\
\text { (2) Location of antenna must be } \\
\text { specified. }\end{array}$ \\
\hline $\begin{array}{l}\text { Time (ToA, } \\
\text { TDoA) }\end{array}$ & $\begin{array}{c}\text { Time } \\
\text { difference of } \\
\text { arrival }\end{array}$ & High & $\begin{array}{l}\text { Good } \\
\text { (Multipath } \\
\text { issues) }\end{array}$ & LOS & Yes & High & $\begin{array}{l}\text { (1) Time synchronization needs. } \\
\text { (2) Location of antenna must be } \\
\text { specified. }\end{array}$ \\
\hline Fingerprinting & $\begin{array}{l}\text { Received } \\
\text { signal } \\
\text { strength }\end{array}$ & High & Good & Both & No & Medium & $\begin{array}{l}\text { (1) Need heavy calibration. } \\
\text { (2) Location of antenna is not } \\
\text { necessary. }\end{array}$ \\
\hline $\begin{array}{l}\text { Dead } \\
\text { reckoning }\end{array}$ & $\begin{array}{l}\text { Acceleration, } \\
\text { velocity }\end{array}$ & $\begin{array}{l}\text { Low to } \\
\text { medium }\end{array}$ & Good & NLOS & Yes & Low & $\begin{array}{l}\text { Inaccuracy of the process is } \\
\text { cumulative, so the deviation in the } \\
\text { position fix grows with time. }\end{array}$ \\
\hline Map matching & $\begin{array}{l}\text { An algorithm } \\
\text { based on } \\
\text { algorithms } \\
\text { based on } \\
\text { projection } \\
\text { and pattern } \\
\text { recognition }\end{array}$ & Medium & $\begin{array}{l}\text { Medium } \\
\text { (indoor) } \\
\text { Good } \\
\text { (outdoor) }\end{array}$ & NLOS & Yes & Medium & $\begin{array}{l}\text { (1) Map matching purely focus on } \\
\text { algorithms and not fully on position } \\
\text { methods, coordinate transformation, } \\
\text { and geocoding. } \\
\text { (2) Using pattern recognition, high } \\
\text { computing complex and poor real time } \\
\text { issue occur. }\end{array}$ \\
\hline
\end{tabular}

TABLE 2: WiFi strengths, weaknesses, and opportunities.

\begin{tabular}{|c|c|}
\hline Strengths & $\begin{array}{l}\text { (i) Found in almost every building, fairly good } \\
\text { available signal strengths. } \\
\text { (ii) WiFi signals are able to penetrate walls in } \\
\text { where GPS fails. } \\
\text { (iii) Targeted location fingerprints available. }\end{array}$ \\
\hline Weaknesses & $\begin{array}{c}\text { (i) Site surveying time consuming and labor } \\
\text { intensive. } \\
\text { (ii) Multipath influenced by presence of } \\
\text { Physical objects. } \\
\text { (iii) Signal strength changes in variations due } \\
\text { to time. } \\
\text { (iv) Interfere possible with other appliances in } \\
\text { the } 2.4 \mathrm{GHz} \text { ISM. }\end{array}$ \\
\hline Opportunities & $\begin{array}{l}\text { (i) Fingerprinting does not need geometric } \\
\text { surveys. } \\
\text { (ii) Fingerprinting only necessary at selected } \\
\text { places. }\end{array}$ \\
\hline
\end{tabular}

be a measurement that includes the information about all stations and their received signal strength (RSS). When the localization system is operational, online phase, the mobile station measures signal properties at unknown spot. Then, the current measured signal strength values are compared for the best agreement with a database (radio map). The major drawback of the fingerprinting approach is the laborious and time-consuming calibration process. Furthermore, adding signal stations would challenge the ease of setup in fingerprinting. Beside this, the main challenge to the techniques based on location fingerprinting is sensitivity to environment changes such as object moving into the building (e.g., people, furniture), diffraction, and reflection, which result in changes in signal propagation. To maintain the positioning accuracy, the calibration process should be periodically repeated to a recalculation of the predefined signal strength map.

Fingerprinting-based positioning algorithms using pattern recognition techniques are deterministic and probabilistic, $K$-nearest-neighbor $(K N N)$, artificial neural networks, Bayesian inference, support vector machine (SVM), or their combinations.

In this part of paper, we are presenting some recent research work done on WiFi localization with a specific focus on fingerprinting-based localization technique.

Different approaches using WiFi access points are studied from time to time. Pereira et al. [36] developed a functional application for a smartphone indoor/outdoor localization system publicly available for download with a name called "Locate Me." It was developed for mobile devices running Android OS and takes advantage of the GPS and WiFi modules to acquire the location of a person. With this system, anybody can find their friends wherever they are. The application sends the current location of the device to the server where it is stored. From that moment on, 
TABLE 3: Comparison of common position systems used for localization.

\begin{tabular}{|c|c|c|c|c|c|c|}
\hline System & Accuracy & $\begin{array}{l}\text { Principles used } \\
\text { for localization }\end{array}$ & Coverage & $\begin{array}{c}\text { Power } \\
\text { consumption }\end{array}$ & Cost & Remarks \\
\hline GPS & $6 \mathrm{~m}-10 \mathrm{~m}$ & ToA & $\begin{array}{l}\text { Good outdoor } \\
\text { Poor indoor }\end{array}$ & Very high & High & $\begin{array}{l}\text { (1) Satellite based } \\
\text { Positioning. } \\
\text { (2) Processing time and } \\
\text { computation is slow. }\end{array}$ \\
\hline Infrared & $1 \mathrm{~m}-2 \mathrm{~m}$ & Proximity, ToA & Good Indoor & Low & Medium & $\begin{array}{l}\text { (1) Short range detection. } \\
\text { (2) No invasion of } \\
\text { multipath. }\end{array}$ \\
\hline WiFi & $1 \mathrm{~m}-5 \mathrm{~m}$ & $\begin{array}{l}\text { Proximity, ToA, } \\
\text { TDoA, RSSI } \\
\text { Fingerprinting, } \\
\text { and RSSI } \\
\text { theoretical } \\
\text { propagation } \\
\text { model } \\
\end{array}$ & $\begin{array}{c}\text { Building level } \\
\text { (outdoor/indoor) }\end{array}$ & High & Low & $\begin{array}{l}\text { (1) Infrastructure available } \\
\text { everywhere. } \\
\text { (2) Initial deployment is } \\
\text { expensive. } \\
\text { (3) Multipath susceptible } \\
\text { slightly. }\end{array}$ \\
\hline Ultrasound & $3 \mathrm{~cm}-1 \mathrm{~m}$ & ToA, AoA & Indoor & Low & Medium & $\begin{array}{l}\text { (1) Sensitive to } \\
\text { environmental. } \\
\text { (2) No invasion of } \\
\text { multipath. }\end{array}$ \\
\hline RFID & $1-2 \mathrm{~m}$ & $\begin{array}{l}\text { Proximity, TOA, } \\
\text { RSSI theoretical } \\
\text { propagation } \\
\text { model }\end{array}$ & Indoor & Low & Low & $\begin{array}{l}\text { (1) Real time location } \\
\text { system. } \\
\text { (2) Response time is high. } \\
\text { (3) Manual programming. }\end{array}$ \\
\hline Bluetooth & $2 m-5 m$ & $\begin{array}{c}\text { RSSI } \\
\text { fingerprinting } \\
\text { and RSSI } \\
\text { theoretical } \\
\text { propagation } \\
\text { model } \\
\end{array}$ & Indoor & Low & High & $\begin{array}{l}\text { (1) Data transfer speed is } \\
\text { high. } \\
\text { (2) Limitation in mobility. }\end{array}$ \\
\hline ZigBee & $3 m-5 m$ & $\begin{array}{l}\text { RSSI } \\
\text { fingerprinting } \\
\text { and RSSI } \\
\text { theoretical } \\
\text { propagation } \\
\text { model } \\
\end{array}$ & Indoor & Low & Low & $\begin{array}{l}\text { (1) Low data transmission } \\
\text { rate. } \\
\text { (2) Nodes are mostly asleep. }\end{array}$ \\
\hline FM & $2 m-4 m$ & $\begin{array}{c}\text { RSSI } \\
\text { fingerprinting }\end{array}$ & Indoor & Low & Low & $\begin{array}{l}\text { (1) Less susceptible to } \\
\text { objects. } \\
\text { (2) Signal is strong; due to } \\
\text { this, it covers large areas. }\end{array}$ \\
\hline
\end{tabular}

$\mathrm{cm}$ : centimeters; m: meters.

all friends can access this position and see it on the map. Google Maps Android API is used to represent the users' location, and it has two views available: road view and satellite view. This location system is based on 4 different methods of localization, three for indoor environments and one for outdoors. The fingerprint localization method is used for indoor location.

Indoor localization using WiFi based fingerprinting and trilateration techniques for LBS application is presented by S. Chan et al. [37]. The paper combined two different WiFi approaches to locate a user in an indoor environment. The first method involves the use of fingerprint matching to compare signal strength data received from nearby access points (AP) by the user, to the reference data stored in the WiFi signal strength database. The second approach uses distance-based trilateration approach using three known AP coordinates detected in the user's device to derive the position. The combination of the two steps enhances the accuracy of the user position in an indoor environment allowing location based services (LBS) such as mobile augmented reality (MAR) to be deployed more effectively in the indoor environment. An improvement is necessary for finding the correct match for the fingerprinting method with help of incorporating certain database correlation algorithms such as $K$-nearest-neighbor or probabilistic like a hidden Markov model.

In [38], the authors design a multifloor indoor positioning system based on Bayesian graphical models (BGM). In this paper, the author first studied the RSS properties that will affect the overall accuracy of our model like a 


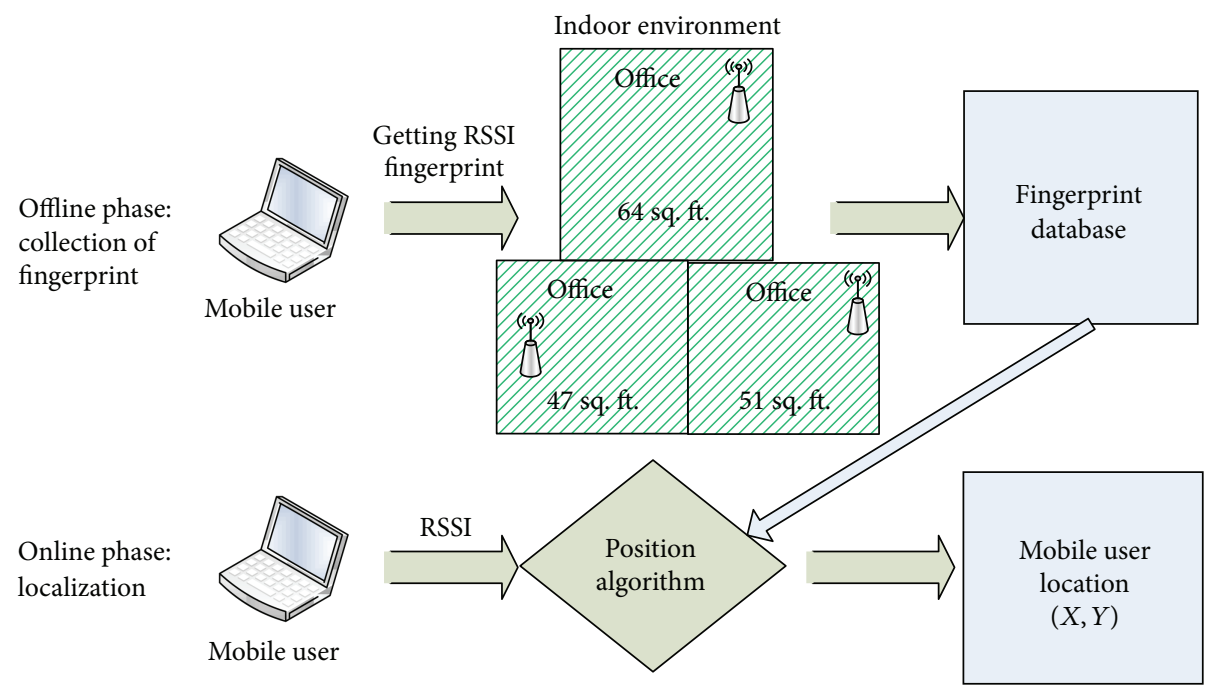

FIGURE 8: Fingerprinting based positioning.

normal distribution of RSS, using RSS in infer location, and multifloor effect. Markov chain Monte Carlo (MCMC) sampling techniques are used. At the last stage, the author tested their model with four sets of MCMC sampling techniques and compared their results with two well-known location determination systems (RADAR and the Horus). The achieved accuracy is $2: 3 \mathrm{~m}$ in a multi-floor environment with a small amount of training points.

By using Wi-Fi, it is possible to define the position of people or assets with good accuracy. In [39], the authors proposed a novel positioning algorithm named predicted $K$-nearest-neighbor $(P K N N)$ which estimates the current position of a mobile user not only by using $K$ found neighbors but also by utilizing its previous positions and speed. In the first stage of the experiment, weighted $K$-nearest neighbors $(W K N N)$ are used for the position of the tag which is to be estimated. In the second stage, prediction is done for the next probable displacement, based on previous user positions and speeds of Wi-Fi tags. The performance of PKNN for indoor positioning has been evaluated by the experimental test bed. By comparison with $K N N$, PKNN performs well by $33 \%$ or at mean 1.3 meter improvement in error.

A novel, information-theoretic approach is presented by Fang and Lin [40] for building a WLAN-based indoor positioning system based on the location fingerprinting system. The proposed technique is based on principal component analysis (PCA) which transforms received signal strength (RSS) into principal components (PCs) such that the information of all access points (APs) is more efficiently utilized. Instead of selecting APs for the positioning which was done by previous researchers, the proposed technique changes the elements with a subset of PCs improvement of accuracy and reduces the online computation. The comparison has been done with AP selection and the Horus system. The proposed approach delivers a significantly improved accuracy. The results show that the mean error is reduced by 33.75 percent and the complexity is decreased by 40 percent.
Received signal strength indication (RSSI) is defined by the IEEE 802.11 Standard. It is a measurement of the $\mathrm{RF}$ energy, and the unit is $\mathrm{dBm}$. Mobile client (MC) can get the RSSI from access point (AP) on the WLAN. The RSSI is decreased exponentially as the distance from AP increased, and this can be expressed by log path loss model. Reference [41] has been presented, using Dominant AP'S RSSI Localization (DARL) algorithms. Using dual log model because of attenuation factor, the parameters are classified into two parts. Firstly, DARL algorithm uses the strongest RSSI from an AP. Secondly, AP trace-back algorithm was suggested as the method for updating the information of unknown AP on the radio map. Optimal filtering system to the proposed algorithm is needed for getting more increased accuracy.

Fingerprinting accuracy performance depends on the number of base stations and the density of calibration points where the fingerprints are taken. Recorded RSSI varies in time, even if there are no changes to the environment. In order to eliminate the deviation of attenuation in the signal, the RSS values are to be averaged over a certain time interval up to several minutes at each fingerprint location. Hansen et al. [42] draw on active user participation relying on the contribution of end users by marking their location on a floor plan while recording the fingerprints. The author concludes from long-term measurements over a period of two months that static radio maps cannot be used for room identification even in modest dynamic environments and therefore recommends dynamically adapting algorithms.

In [43], the author presents the design, implementation, and evaluation of a fine-grained indoor fingerprinting system (FIFS) as shown in Figure 5. Data are modulated on multiple subcarriers at different frequencies and transmitted simultaneously in Orthogonal frequency division multiplexing $(\mathrm{OFDM})$. The author takes the value that estimates the channel in each subcarrier called channel state information (CSI). Different from RSSI, CSI is a fine-grained value from 
the PHY layer which describes the amplitude and phase on each subcarrier in the frequency domain. To obtain high accuracy and low complexity of indoor localization, the FIFS was comprised of two parts: first, the process of raw CSI values of measurement by integrating frequency diversity (amplitudes and phases at multiple propagation paths) and spatial diversity of location using multiple antennas, and then it builds up a radio map; second, determining the position of objects by correlation calculation augmented with a probability algorithm. An experiment is done in two typical indoor scenarios with commercial IEEE 802.11 NICs. The median accuracy of $0: 65 \mathrm{~m}$ is improved compared with the RSS-based Horus system 0.2 with gain of about $24 \%$.

Aboodi and Tat-Chee [44] proposed an indoor positioning algorithm called WiFi-based indoor (WBI) positioning algorithm. WBI-based on WiFi received signal strength (RSS) technology in conjunction with trilateration techniques. The WBI algorithm estimates the location using RSS values previously collected from within the area of interest using LSE algorithm, determines whether it falls within the MinMax bounding box, corrects for nonline-of-sight propagation effects on positioning errors using Kalman filtering, and finally updates the location estimation using least square estimation (LSE). The paper analyzed the complexity of the proposed algorithm and compares its performance against existing algorithms. Furthermore, the proposed WBI algorithm achieves an average accuracy of $2.6 \mathrm{~m}$.

The authors of [45] proposed a GPS-Like zeroconfiguration indoor positioning system based on received signal strength (RSS) of the popular WiFi network as shown in Figure 9. The proposed system does not require a time-consuming offline radio survey prior knowledge about the area or new hardware unlike current RSS-based indoor systems. Similar to GPS, the proposed system consists of three sections as shown in Figure 9: network segment (WiFi), control segment, and user segment. Between network segment and control segment, RSS observations are exchanged periodically. The control segment uses a novel hybrid propagation modeling (PM) technique using logarithmic decay model augmented by a nonlinear Gaussian process regression (GPR) that models RSS residuals that cannot be modeled by the traditional logarithmic decay models indoors. The proposed system provides $2-3 \mathrm{~m}$ accuracy in indoor environments.

Yang et al. [46] proposed a wireless indoor localization approach called Locating in fingerprint space (LiFS). In fingerprinting method, radio base requires a process of site survey, in which radio signatures of an interested area are marked with their real recorded locations. Site survey involves intensive costs on manpower and time and is vulnerable to environmental dynamics. The author investigates the sensors integrated in modern mobile phones and user motions to construct the radio map of a floor plan, which is previously obtained only by site survey. On this basis, they design LiFS, an indoor localization system based on off-theshelf WiFi infrastructure and mobile phones. An experiment was performed in an office building covering over $1600 \mathrm{~m}^{2}$, and the results show that LiFS achieves low human cost, rapid system deployment.

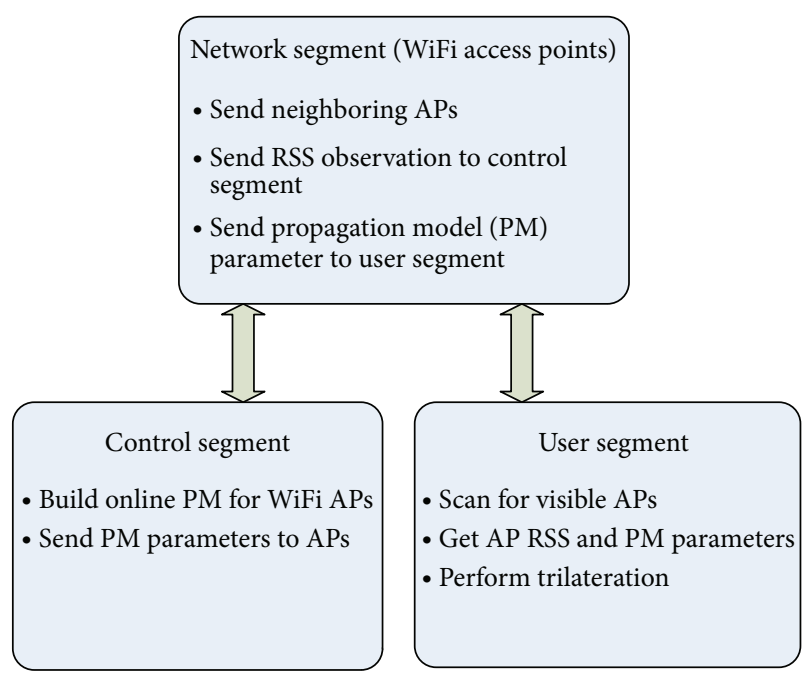

FIGURE 9: Simplified block diagram [45].

IEEE $802.11 \mathrm{n}$ is an amendment to the IEEE 802.112007 wireless networking standard to improve network throughput over the two previous standards-802.11a and $802.11 \mathrm{~g}$ - with a significant increase in the maximum net data rate from $54 \mathrm{Mbit} / \mathrm{s}$ to $600 \mathrm{Mbit} / \mathrm{s}$. It also improves WLAN standard in terms of network throughput by adding a technology which supports multiple antenna configurations, known as multiple-input multiple-output (MIMO). Xiong and Jamieson [47] presented ArrayTrack, an indoor location system that uses angle-of-arrival techniques to locate wireless clients indoors in a wireless local area network. ArrayTrack combines best of breed algorithms for AoA based direction estimation and spatial smoothing with novel algorithms for suppressing non-line-of-sight reactions that occur frequently indoors. Based on simulation, ArrayTrack achieved median $25 \mathrm{~cm}$ location accuracy when clients are stationary indoors.

\section{Conclusion and Future Trends}

This paper surveys the recent advances in wireless indoor localization techniques and system. Different technological solutions for wireless indoor positioning and navigation are discussed, and several tradeoffs among them are observed. Regardless of the plenty of approaches which exist to handle the indoor positioning problem, current solutions cannot cope with the performance level that significant applications required. In short, requirements for different application environments are accuracy/precision, coverage, availability, and minimal costs for local installations. To achieve this shortcoming, a good portion of research approaches is required to handle these challenges. Some of the future trends of wireless indoor positioning systems are as follows:

(1) new hybrid solution for positioning and tracking estimation in $4 \mathrm{G}$ with the currently available position system,

(2) need of cooperative mobile localization which will help mobile nodes among each other to determine their locations, 
(3) new innovative applications for mobile in which location information can be used to improve the quality of users' experience and to add value to existing services offered by wireless providers.

\section{Acknowledgments}

The authors would like to thank the National University of Malaysia and the Ministry of Science, Technology and Innovation for the financial support of this work, under the Grant Ref. 01-01-02-SF0788. The authors also would like to thank the anonymous reviewers for their valuable feedback.

\section{References}

[1] Y. Liu and Z. Yang, Location, Localization, and Localizability. Location-awareness Technology for Wireless Networks, Springer, 2010.

[2] D. Mohapatra and S. B. Suma, "Survey of location based wireless services," in Proceedings of the 7th IEEE International Conference on Personal Wireless Communications (ICPWC '05), pp. 358362, January 2005.

[3] H. Liu, H. Darabi, P. Banerjee, and J. Liu, "Survey of wireless indoor positioning techniques and systems," IEEE Transactions on Systems, Man and Cybernetics Part C, vol. 37, no. 6, pp. 10671080, 2007.

[4] Y. Gu, A. Lo, and I. Niemegeers, "A survey of indoor positioning systems for wireless personal networks," IEEE Communications Surveys and Tutorials, vol. 11, no. 1, pp. 13-32, 2009.

[5] D. Zhang, F. Xia, Z. Yang, L. Yao, and W. Zhao, "Localization technologies for indoor human tracking," in Proceedings of the 5th International Conference on Future Information Technology (FutureTech '10), May 2010.

[6] J. Yiming, Indoor Location Determination, in Location-Based Services Handbook, CRC Press, 2010.

[7] X. Hu, L. Cheng, and G. Zhang, "A Zigbee-based localization algorithm for indoor environments," in Proceedings of the International Conference on Computer Science and Network Technology (ICCSNT '11), pp. 1776-1781, December 2011.

[8] F. Seco, A. R. Jiménez, C. Prieto, J. Roa, and K. Koutsou, "A survey of mathematical methods for indoor localization," in Proceedings of the 6th IEEE International Symposium on Intelligent Signal Processing (WISP '09), pp. 9-14, August 2009.

[9] M. Vossiek, L. Wiebking, M. Glänzer, D. Mastela, and M. Christmann, "Wireless local positioning-concepts, solutions, applications," in Proceedings of the IEEE Radio and Wireless Conference (RAWCON '03), pp. 219-224, August 2003.

[10] Cisco System, Wi-Fi Location-Based Services 4.1 Design Guide, 2008.

[11] M. B. Ismail, A. F. A. Boud, and W. N. W. Ibrahim, "Implementation of location determination in a wireless local area network (WLAN) environment," in Proceedings of the 10th International Conference on Advanced Communication Technology (ICACT '34), pp. 894-899, February 2008.

[12] S. Gezici, "A survey on wireless position estimation," Wireless Personal Communications, vol. 44, no. 3, pp. 263-282, 2008.

[13] H. Samuel, S. Connell, I. Milligan, D. Austin, T. L. Hayes, and P. Chiang, "Indoor localization using pedestrian dead reckoning updated with RFID-based fiducials," in Proceedings of the 33rd Annual International Conference of the IEEE Engineering in Medicine and Biology Society (EMBS '11), pp. 7598-7601, September 2011.

[14] D. Pai, M. Malpani, I. Sasi, N. Aggarwal, and P. S. Mantripragada, "A Robust pedestrian dead reckoning system on smartphones," in Proceedings of the IEEE 11th International Conference on Trust, Security and Privacy in Computing and Communications (TrustCom '12), pp. 2000-2007, June 2012.

[15] M. A. Quddus, W. Y. Ochieng, and R. B. Noland, "Integrity of map-matching algorithms," Transportation Research Part C, vol. 14, no. 4, pp. 283-302, 2006.

[16] M. Attia, A. Moussa, X. Zhao, and N. El-Sheimy, "Assisting personal positioning in indoor environments using map matching," Archives of Photogrammetry, Cartography and Remote Sensing, vol. 22, pp. 39-49, 2011.

[17] A. M. Ahmed, W. Zhu, and T. M. Bekele, "Map-matching and positioning uncertainty in Location Based Services (LBS)," in Proceedings of the International Conference on Asia Agriculture and Animal, Singapoore, 2011.

[18] 2013, http://en.wikipedia.org/wiki/Global_Positioning_System.

[19] J. Xiao, Z. Liu, Y. Yang, D. Liu, and H. Xu, "Comparison and analysis of indoor wireless positioning techniques," in Proceedings of the International Conference on Computer Science and Service System (CSSS '11), pp. 293-296, June 2011.

[20] R. Casas, D. Cuartielles, Á. Marco, H. J. Gracia, and J. L. Falcó, "Hidden issues in deploying an indoor location system," IEEE Pervasive Computing, vol. 6, no. 2, pp. 62-69, 2007.

[21] P. Vorst, J. Sommer, C. Hoene et al., "Indoor positioning via three different RF technologies," in Proceedings of the 4th European Workshop on RFID Systems and Technologies (RFID SysTech '08), pp. 1-10, June 2008.

[22] P. Bahl and V. N. Padmanabhan, "RADAR: an in-building RFbased user location and tracking system," in Proceedings of the 19th Annual Joint Conference of the IEEE Computer and Communications Societies, pp. 775-784, March 2000.

[23] F. Subhan, H. Hasbullah, A. Rozyyev, and S. T. Bakhsh, "Indoor positioning in Bluetooth networks using fingerprinting and lateration approach," in Proceedings of the International Conference on Information Science and Applications (ICISA '11), April 2011.

[24] H. J. Perez Iglesias, V. Barral, and C. J. Escudero, "Indoor person localization system through RSSI Bluetooth fingerprinting," in Proceedings of the 19th International Conference on Systems, Signals and Image Processing (IWSSIP '12), pp. 40-43, April 2012.

[25] Z. Li, W. Dehaene, and G. Gielen, "System design for ultralow-power UWB-based indoor localization," in Proceedings of the IEEE International Conference on Ultra-Wideband (ICUWB '07), pp. 580-585, September 2007.

[26] A. Popleteev, V. Osmani, and O. Mayora, "Investigation of indoor localization with ambient FM radio stations," in Proceedings of the IEEE International Conference on Pervasive Computing and Communications (PerCom '12), 2012.

[27] V. Moghtadaiee, A. G. Dempster, and S. Lim, "Indoor localization using FM radio signals: a fingerprinting approach," in Proceedings of the International Conference on Indoor Positioning and Indoor Navigation (IPIN '11), September 2011.

[28] ZigBee Technology, 2013, http://www.zigbee.org/About/ AboutTechnology/ZigBeeTechnology.aspx.

[29] S. Fernández, D. Gualda, J. C. García, J. J. García, J. Ureña, and R. Gutiérrez, "Indoor location system based on ZigBee devices and metric description graphs," in Proceedings of the 7th IEEE International Symposium on Intelligent Signal Processing (WISP '11), pp. 4-8, September 2011. 
[30] S. Zirazi, P. Canalda, H. Mabed, and F. Spies, "Wi-Fi access point placement within stand-alone, hybrid and combined wireless positioning systems," in Proceedings of the 4th International Conference on Communications and Electronics (ICCE '12), pp. 279-284, 2012.

[31] H. Mehmood and N. K. Tripathi, Hybrid Positioning Systems: A Review, LAP LAMBERT Academic Publishing, 2011.

[32] A. Runge, M. Baunach, and R. Kolla, "Precise self-calibration of ultrasound based indoor localization systems," in Proceedings of the International Conference on Indoor Positioning and Indoor Navigation (IPIN '11), September 2011.

[33] C. Medina, J. C. Segura, and S. Holm, "Feasibility of ultrasound positioning based on signal strength," in Proceedings of the International Conference on Indoor Positioning and Indoor Navigation (IPIN '12), pp. 1-9, November 2012.

[34] C. Marc, M. E. Israel, and B. A. Francisco, "Location in wireless local area networks," in Location-Based Services Handbook, pp. 67-90, CRC Press, 2010.

[35] M. A. Youssef, A. Agrawala, and A. U. Shankar, "WLAN location determination via clustering and probability distributions," in Proceedings of the 1st IEEE International Conference on Pervasive Computing and Communications (PerCom '03), pp. 143-150, March 2003.

[36] C. Pereira, L. Guenda, and B. N. Carvalho, "A smart-phone indoor/outdoor localization system," in Proceedings of the International Conference on Indoor Positioning and Indoor Navigation (IPIN '11), 2011.

[37] S. Chan and G. Sohn, "Indoor localization using Wi-Fi based fingerprinting and trilateration techiques for LBS applications," in Proceedings of the 7th International Conference on 3D Geoinformation, Quebec, Canada, May 2012.

[38] A. S. Al-Ahmadi, A. I. Omer, M. R. Kamarudin, and T. A. Rahman, "Multi-floor indoor positioning system using bayesian graphical models," Progress In Electromagnetics Research B, vol. 25, pp. 241-259, 2010.

[39] S. Khodayari, M. Maleki, and E. Hamedi, "A RSS-based fingerprinting method for positioning based on historical data," in Proceedings of the International Symposium on Performance Evaluation of Computer and Telecommunication Systems (SPECTS '2010), pp. 306-310, July 2010.

[40] S.-H. Fang and T. Lin, "Principal component localization in indoor wlan environments," IEEE Transactions on Mobile Computing, vol. 11, no. 1, pp. 100-110, 2012.

[41] P. DoWoo and P. Joon Goo, "An enhanced ranging scheme using WiFi RSSI measurements for ubiquitous location," in Proceedings of the 1st ACIS/JNU International Conference on Computers, Networks, Systems and Industrial Engineering (CNSI '11), 2011.

[42] R. Hansen, R. Wind, C. S. Jensen, and B. Thomsen, "Algorithmic strategies for adapting to environmental changes in 802.11 location fingerprinting," in Proceedings of the International Conference on Indoor Positioning and Indoor Navigation (IPIN '10), Zurich, Switzerland, September 2010.

[43] J. Xiao, K. Wu, Y. Yi, and L. M. Ni, "FIFS: fine-grained indoor fingerprinting system," in Proceedings of the 21st International Conference on Computer Communications and Networks (ICCCN '12), 2012.

[44] A. Aboodi and W. Tat-Chee, "Evaluation of WiFi-based indoor (WBI) positioning algorithm," in Proceedings of the 3rd FTRA International Conference on Mobile, Ubiquitous, and Intelligent Computing (MUSIC '12), pp. 260-264, June 2012.
[45] M. M. Atia, M. Korenberg, and A. Noureldin, "A consistent zero-configuration GPS-Like indoor positioning system based on signal strength in IEEE 802.11 networks," in Proceedings of the IEEE/ION Position Location and Navigation Symposium (PLANS '12), pp. 1068-1073, April 2012.

[46] Z. Yang, C. Wu, and Y. Liu, "Locating in fingerprint space: wireless indoor localization with little human intervention," in Proceedings of the 18th Annual International Conference on Mobile Computing and Networking (Mobicom '12), pp. 269-280, 2012.

[47] J. Xiong and K. Jamieson, ArrayTrack: A Fine-Grained Indoor Location System, ACM HotMobile, 2012. 

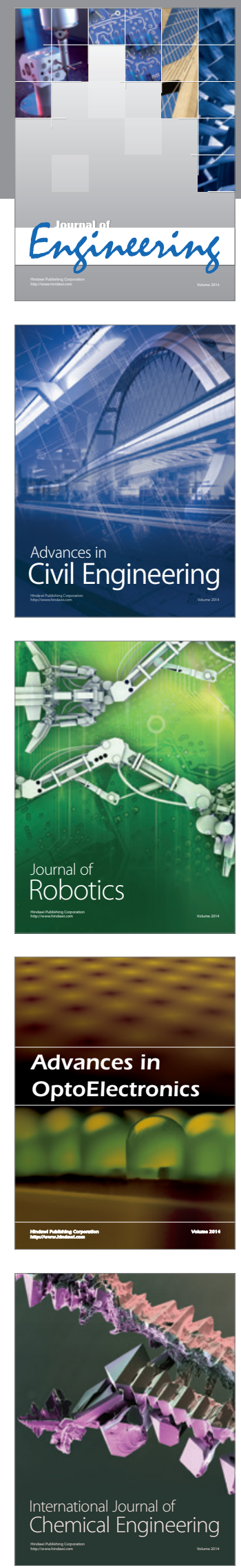

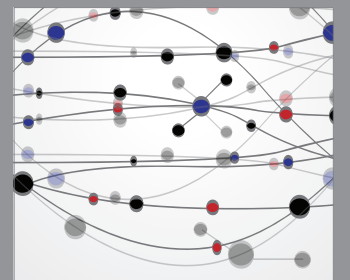

The Scientific World Journal
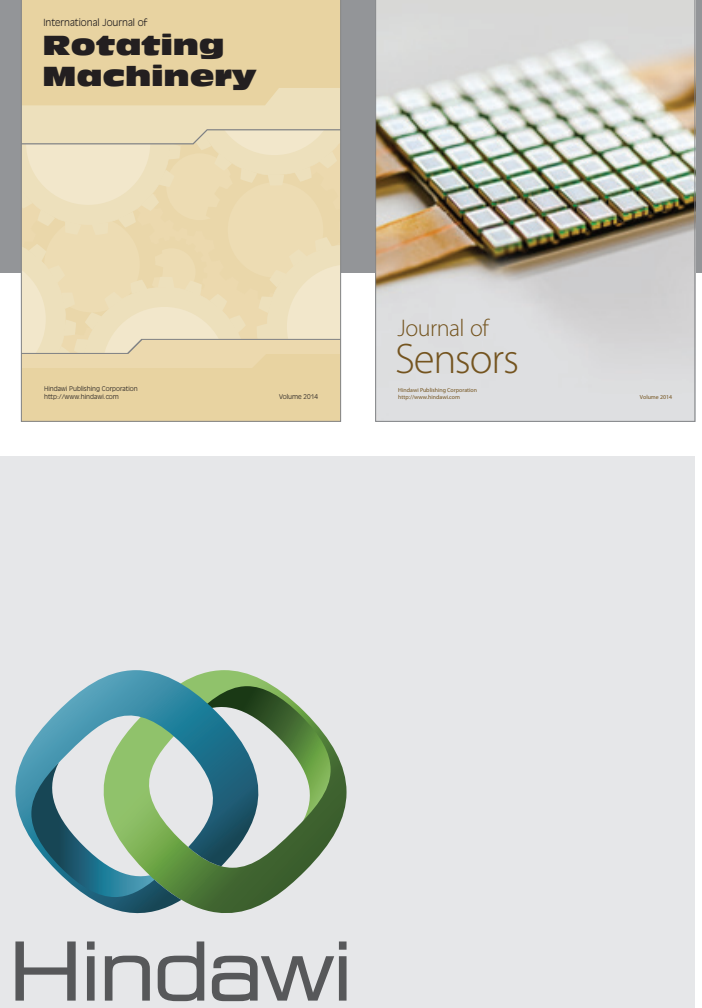

Submit your manuscripts at http://www.hindawi.com
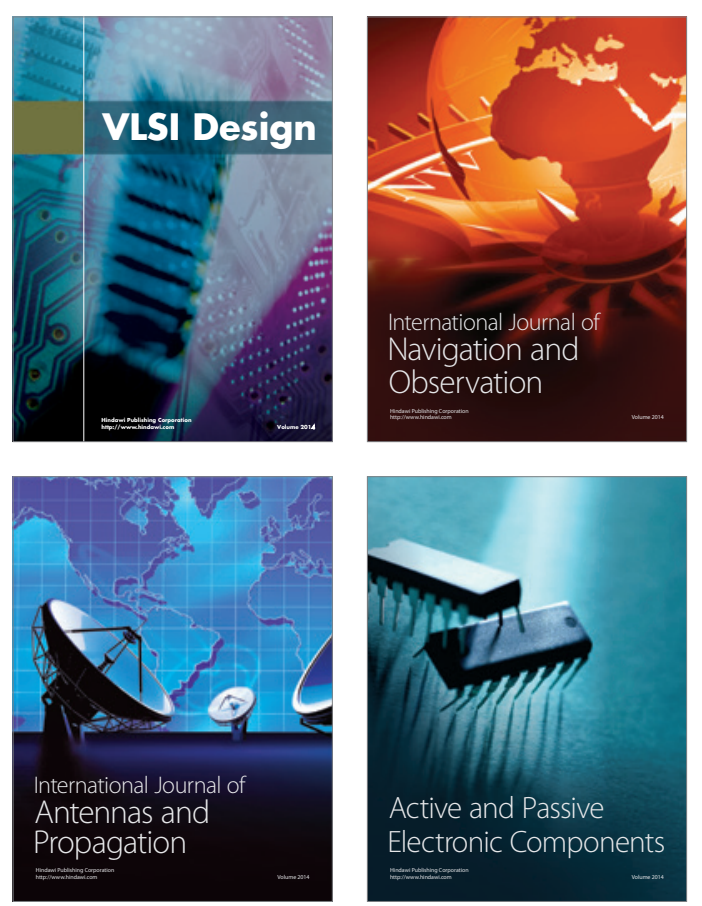
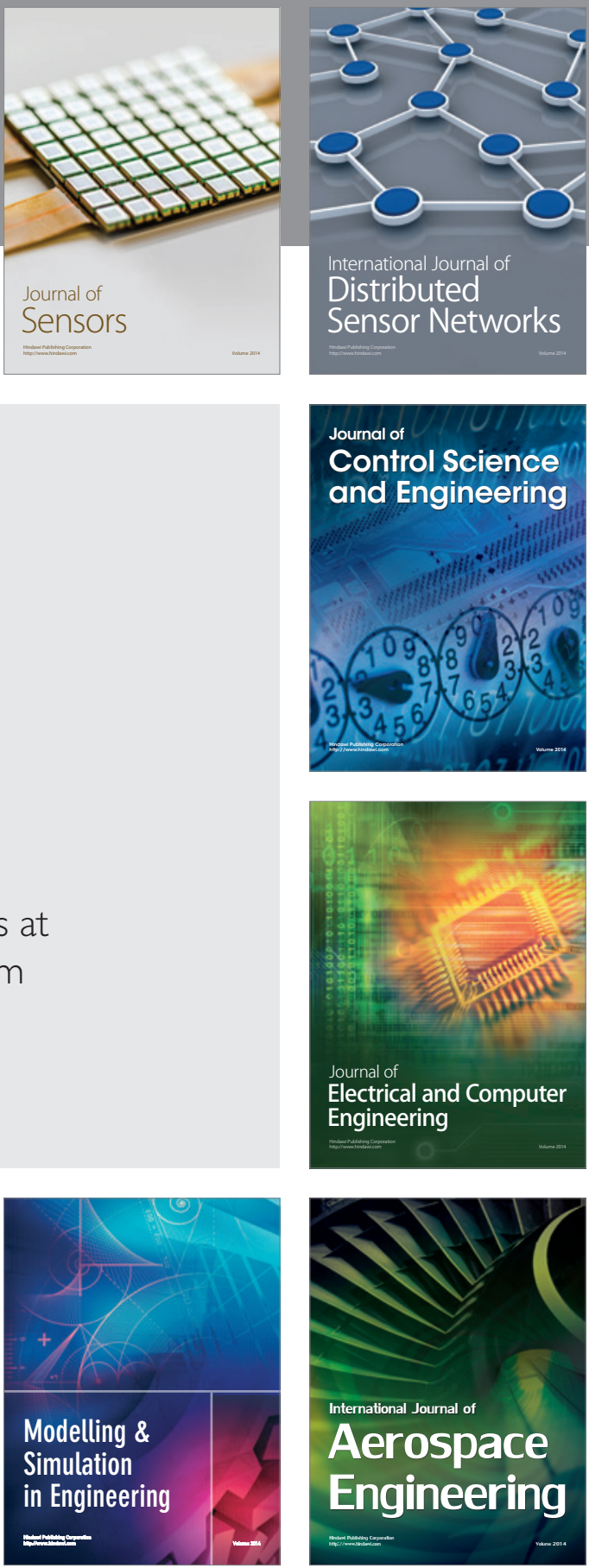

Journal of

Control Science

and Engineering
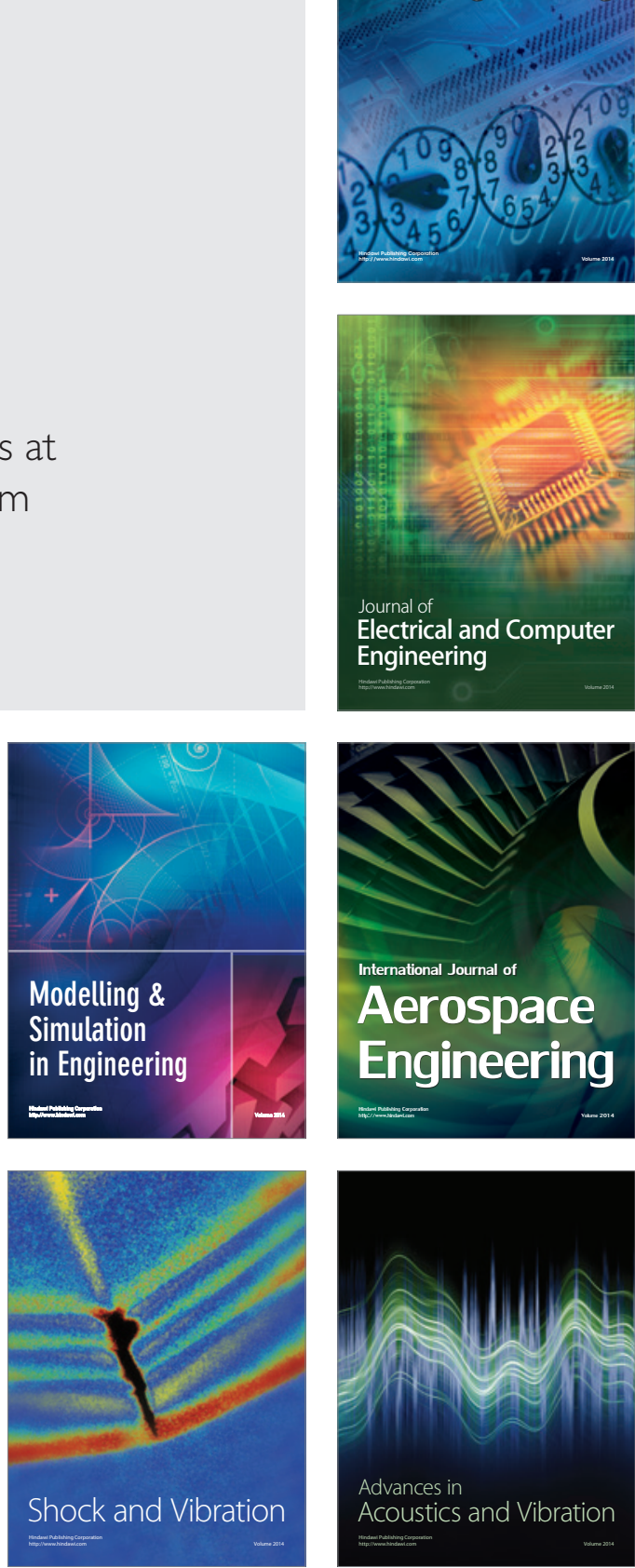